INPLASY

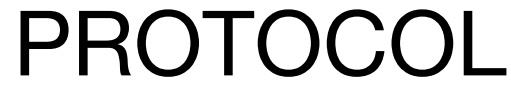

To cite: Alalqam et al.

Clinician-Reported Barriers to the Provision of Brief Advice

for Drug Use in Hospital

Inpatient Settings. Inplasy

protocol 202120035. doi:

10.37766/inplasy2021.2.0035

Received: 09 February 2021

Published: 09 February 2021

Corresponding author:

Razi Alalqam

razialalqam@rcsi.com

Author Affiliation:

Royal College of Surgeons in Ireland (RCSI)

Support: RCSI.

Review Stage at time of this submission: The review has not yet started.

Conflicts of interest:

None declared.

\section{Clinician-Reported Barriers to the Provision of Brief Advice for Drug Use in Hospital Inpatient Settings}

\author{
Alalqam, R¹; Al Mashhad, $\mathrm{H}^{2}$; Doyle, F. ${ }^{3}$
}

Review question / Objective: What are the barriers to the provision of brief advice for drug use in hospital inpatient settings?

Condition being studied: Clinician-reported barriers to the provision of brief advice for drug use in hospital inpatient settings.

Information sources: We will search Scopus, MEDLINE, PsycINFO and CINAHL for English language articles with no limitations in the publication dates or study types. After that, Google Scholar will be used as citation databases to identify any missed research articles.

INPLASY registration number: This protocol was registered with the International Platform of Registered Systematic Review and Meta-Analysis Protocols (INPLASY) on 09 February 2021 and was last updated on 09 February 2021 (registration number INPLASY202120035).

\section{INTRODUCTION}

Review question / Objective: What are the barriers to the provision of brief advice for drug use in hospital inpatient settings?

Condition being studied: Clinician-reported barriers to the provision of brief advice for drug use in hospital inpatient settings.

\section{METHODS}

Search strategy: Four online databases will be searched: Scopus, Medline, PsycINFO and CINHAL. Any full-text article in the English language will be considered, with no limitations on the study type. The following searching terms will be used: 
"Physicians", "barriers", "Drug users", "Hospital" and their synonyms.

Participant or population: Hospital inpatients and those in emergency departments who may not be admitted as inpatients.

Intervention: Clinician-reported barriers to the provision of brief advice for drug use in hospital inpatient settings.

\section{Comparator: None.}

Study designs to be included: No limitations in the study designs included.

Eligibility criteria: Full-text articles from any study type that reports on the barriers to the provision of brief advice for drug use in hospital inpatient settings will be included. The exclusion criteria is non-English language, non-hospital settings, and articles that are not full text.

Information sources: We will search Scopus, MEDLINE, PsycINFO and CINAHL for English language articles with no limitations in the publication dates or study types. After that, Google Scholar will be used as citation databases to identify any missed research articles.

Main outcome(s): Clinician-reported barriers to the provision of brief advice for drug use in hospital inpatient settings.

Data management: Endnote will be used to manage the citations.

Quality assessment / Risk of bias analysis: CCAT assesses the section of each study, which includes Preliminaries, Introduction, Design, Sampling, Data Collection, Ethics, Results, and Discussion. Two reviewers assess the overall result of each study individually and then discuss their results. Any disagreements will be resolved among themselves or a third reviewer if necessary. Furthermore, we will use the GRADECERQual approach to assess the confidence in the evidence. This approach will help evaluate the barriers mentioned in the studies cited and whether they are a reasonable representation of the phenomena of interest. This approach consists of four components, methodological and limitations component, coherence component, adequacy component and relevance component. When a minimum of 5 studies are gathered, narrative synthesis will be carried. The reported barriers will be combined under three headings of the (COM-B) model which are capability, opportunity and motivation for behaviour. Two authors will report the barriers from each study. They will also count the barriers under each COM-B heading. The final result will comprise of lists of the most common reported barriers in the provision of drug use advice in hospital inpatient settings in conjunction with the COM-B model, which will help frame a formal method for future interventions.

Strategy of data synthesis: Any published research article (qualitative or quantitative) will be included in a narrative synthesis. This will be done when a minimum of 5 studies are gathered. Two authors will report the barriers from each study and categorize them into COM-B model headings which are capability, opportunity and motivation for behaviour. The results will be presented as a narrative summary in a table (e.g. 4 studies reported lack of time; 5 studies reported lack of motivation, etc.). Furthermore, a count of the barriers will be done to present a ranking of the most commonly reported barriers under each COM-B heading, which will help frame a formal method for future interventions. No statistical software is needed to carry these methods. This method of narrative synthesis follows that of Sharpe et al. , 2018 (Sharpe T, Alsahlanee A, Ward KD, Doyle F. Systematic review of clinicianreported barriers to provision of smoking cessation interventions in hospital inpatient settings. Journal of Smoking Cessation. 2018 Dec;13(4):233-43. doi:10.1017/ jsc.2017.25) and Gargaritano et al. , 2020 (Gargaritano KL, Murphy C, Auyeung AB, Doyle F. Systematic Review of ClinicianReported Barriers to Provision of Brief Advice for Alcohol Intake in Hospital Inpatient and Emergency Settings. 
Alcoholism: Clinical and Experimental Research. 2020 Dec;44(12):2386-400. doi:10.1111/acer.14491).

Subgroup analysis: We will carry subgroup analysis based on the healthcare providers, methods and reported barriers similar to Gargaritano et al (2020).

Sensitivity analysis: The COM-B model will be used to categorize reported barriers. Studies reporting a single barrier or stratifying results by staff type, sample size, or study type (quantitative/qualitative) will be excluded from the disregarded similar to Gargaritano et al (2020).

Language: Only studies in the English language will be considered.

Country(ies) involved: Ireland.

Keywords: Physicians; barriers; Drug users; Hospital.

Contributions of each author:

Author 1 - Razi Alalqam.

Email: razialalqam@rcsi.com

Author 2 - Hussain Al Mashhad.

Email: hussainalmashhad@rcsi.com

Author 3 - Frank Doyle.

Email: fdoyle4@rcsi.com 